\title{
Technical Thinking Systems are on the Threshold of Our Society
}

\author{
A.I.Timofeev \\ JVC National Institute of Aviation Technologies, Russia
}

Copyright (C) 2015 by authors, all rights reserved. Authors agree that this article remains permanently open access under the terms of the Creative Commons Attribution License 4.0 International License

\begin{abstract}
The last years much attention is paid to Systems Thinking including international special conferences [1] which presented innovative papers on the theory and its application - Technical Thinking Systems including also a variety of "smart" systems, for example, "smart " cities of future, "smart" homes, "smart" streets and "smart" apartments, "smart" eyes, and "intelligent" hands and so on, discussed the roles of these systems in natural and extreme conditions, computer science, pedagogy, the specific requirements for citizens of future smart cities, the problems of variety of simulations of Systems Thinking. The main feature of the example of the Thinking Systems is the presence of feedback by simulation of physical situations in the system "Brush-Object" (B-O) of manipulation robotics as the result of simulation of spatial sense of touch of human hand at semantic level. Cardinal decision of actual and unsolved Problem of manipulation robotics-capture reliability of non-oriented complex shape objects - is based on application of semiotic structure of relations of contact points as the symbiosis of informatiology, discovering geometrical multi-agent foreshortening of unknown initial situation, and semiotics, discovering semantic component of information. The application of methods and principles of Thinking Systems in robotics can stimulate widespread use of manipulation robotics in previously inaccessible areas of employment rights.
\end{abstract}

Keywords Thinking Systems, Simulation, Methods of Organization, Robotics

\section{Terminology and Some Features of Thinking Systems}

Russian Academician A.N. Kolmogorov considered [2] that simulation of methods of organization (as a set of information processes or actions, coming to expected results) of material systems (including biological) is to use other material elements (and useful algorithms of functioning and elements of structures) to create new systems with essentially the same organization (and their methods) like the original system. Therefore, to his opinion, the quite complete model of a living system should be called as a living system, and the quite complete model of a thinking system should be called as a thinking system.

In general it is known that any smart system can use the principle of selection (by adopted criteria) the solution or need algorithm of limited actions from previously performed their list.

Decision taking procedure of Thinking Systems is implemented by another way.

First of all, some psychologists have considered that usually any normal man try to get ahead the semantics of his future actions (with expected the results) before realization of these actions, otherwise his life will be meaningless.

Therefore Thinking Systems as the original systems of simulation of human informational processes of decision taking, cannot contain ahead the list of solutions with specific limited algorithms of actions, for example, in natural indeterminate environment and so include the following features at autonomous level:

- determination of environment (situation) with its simulation at semantic level,

- cognitive analysis of received information model of environment,

- estimation of received informational model by comparison with the model of expected purpose at parametric level,

- prognosis the results of virtual actions of taken solutions with possible application of self-organization processes,

- generation of adequate solutions and specific algorithms of actions.

So the ability of Thinking Systems to identify and represent the semantics of external environment adequately to semantics of solving tasks at parametric levels autonomously is a direct step from primitive level of thinking to professional level of thinking of Technical intellectual Systems.

\section{One of Actual and Unsolved Problem of Manipulation Robotics}


For example, the long absence of decision of one of actual unsolved Problems of manipulation robotics and prosthetic devices - capture reliability of non - oriented complex shape objects - as the necessary stage of any object's manipulation in natural indeterminate surroundings has a negative impact on the expansion of using of manipulation robotics and prosthesis in Economy, Medicine, natural and extreme conditions for living.

The main reason for existence of this problem is connected with the absence of active force projections of weight of any object in the new multitude of contact points (at the stage before object's separation from the initial position), and obligatory appearance of these active forces later as well as appearance of other passive forces in these contact points during at the stage of object's manipulation due to objective physical laws on our planet (physical problem).

This generates paradoxical situation when at first it is necessary to take decision (by any man or robot) on capture reliability in initial conditions (before separation of object) but then the realization of that decision takes place in another conditions

So the physical situations in the system of the system "B-O" can take different uncontrolled force and moment equilibrium state (stable, unstable, etc.) because of lack of information support.

If adaptation to unknown complex forms (topological task) can be achieved by application any vision system and adaptive robotic gripper, now the problem of capture reliability (physical task) for mentioned reasons has not comprehensive solution in the World.

Therefore manipulation possibilities of any robotics connected with capture of object, can be realized successfully only in the shot frames of beforehand created or

\section{Semantic net of relations}
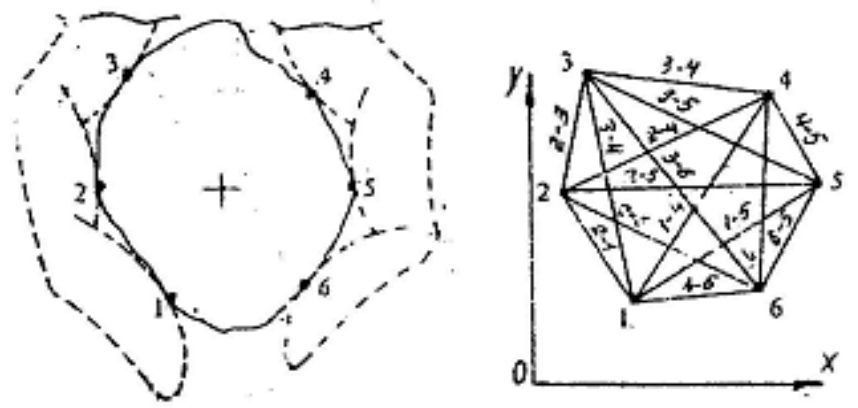

estimated by man determinate conditions.

This also deprives robotics the possibility of any autonomy in different natural situations.

At the present the condition of successful introduction of manipulation robotics in industry is the application of the principle "Forced ordered surroundings" in the form of using of the accessories of orientation, storage and timely delivery of each object in the capture position.

If it is necessary to chance the articles of objects at that case the accessories and grippers of robotics can be the subjects for repair or compensation.

In extreme conditions (space, nuclear industry, oceans, dangerous situations, etc) the operators of manipulators with manual control, deprived of the possibility of using their own biological function of capture (including the spatial sense of touch hand) and so are forced to take decision on capture any object (as the physical task) by using their own view or limited information of force-sensed manipulators.

\section{Cardinal Solution of the Problem}

Cardinal solution [3],[4],[5],[6] of mentioned Problem is based on simulation the only cardinal solution implemented by the Nature on human hand, and is associated with the use of purposefully formalized interdisciplinary knowledge and results of the experiment of revelation of functional principles of motor activity of human hands [6].

Quantitative and qualitative assessments of physical situations with prognosis of reliability of capture are the basis for making appropriate adequate solutions on capture reliability of non-oriented objects (before its separation from the initial position) can be seen at Fig.1 and formula 1:

\section{Structure of F.V.D.O. F.V.D.O.}

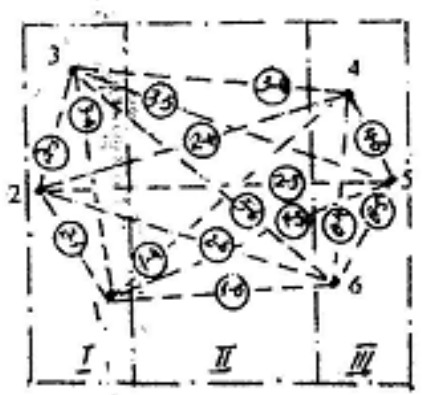

Figure 1. Necessary stages of bionic approach of simulation of organization methods for Thinking Systems (F.V.D.O. - the image of fixed virtual displacements of any object, 1-6-contact points and their relations ). 


\subsection{Prognosis of Capture Reliability of Non-oriented Complex Form Objects}

$\Phi_{\bar{\omega}}^{N}-[\Phi] \Rightarrow \Delta \Phi_{\bar{\omega}}^{N} \Rightarrow \operatorname{sign} \Delta \Phi_{\bar{\alpha}}\left|\begin{array}{c}\oplus \Delta \Phi>0 \\ \oplus \Delta \Phi=0 \\ \oplus \Delta \Phi<0\end{array}\right| \Rightarrow \Pi P^{\circ}$

$\Phi_{\text {об }}{ }^{\mathrm{N}}-$ Factual image F.V.D.O.,

$N$--- Quantity of relations of contact points,

[Ф] - Image F.V.D.O. with permissible parameters,

$\Pi \mathrm{P}^{\mathrm{O}}$ - Prognosis of capture reliability.

The use of internal image language (based on the semiotic structure of relations of contact points) for the purpose of intellectualization of information processes (receiving, processing, storage and transmission of information) in the system "Brush - Object" provides semantic expressiveness as these processes and so parameters of taken decisions with the ability to ensure their endo-physical properties..

Achieved the analogy of the structure of the "inner word" of internal image language with the structure of the "inner word" of human language is the basis of identity (towards biological prototype) of accepted method of organization of information processes as determination of physical situations in the system "B-O" and prognosis capture reliability of non-oriented objects with estimations of physical situations, decision taking and the ability to control physical situations (in case of negative results of prognosis) by using self-organization processes.

The additional difficulties of research of cardinal decision of mentioned problem are connected also with the fact that much of information processes of any human taking decision are on the subconscious level (psychological problem).

The solution contains the determination of physical situations in the system "B- O" with their simulation and cognitive analysis, as well as the geometrization of physical situations, with translation of original information from its "closed" status in "open" status for our consciousness.

.... Quantitative and qualitative assessments of physical situations with prognosis of reliability of capture are the basis for making appropriate adequate solutions on capture reliability of non-oriented objects (before its separation from the initial position).

The use of internal image language (based on the semiotic structure of relations of contact points) for the purpose of intellectualization of information processes (receiving, processing, storage and transmission of information) in the system "B- O" provides semantic expressiveness as these processes and so parameters of taken decisions with the ability to ensure their endo-physical properties..

Some functional principles of movement actions of human hand in indeterminate situations and unity of structures of words or informational units of image language of robot (RL) and natural human language (HL) are the objects of simulation methods of organization processes of human activities for cardinal decision of the problem and for thinking processes.
The adequacy of taken decision is evaluated by the results of prognosis of its virtual actions.

In our case the simulation of methods of organization of human hand's activities as the set of informational processes is able to generate the organization of targeted relations between contact points as the components of structure of new created model (as a feedback of any physical situations in the system "B - O").

The heart of the methodology of creation of mentioned feedback is application of semiotic structure of relations of contact points as the symbiosis of informatiology discovering geometrical multi-agent perspective of any physical situations in the system "B - O" and of semiotics discovering its semantics.

Above provides the semiosis as the sign informational process of formation of capture reliability prognosis with internal non-verbal image language and image F.V.D.O.

Mentioned above must be based on expedient functional principles of movement actions of human hand in indeterminate situations identified experimentally in the similar conditions of these activities.

The successful application of functional principles of human motor hand activities in our case has been taken as the criteria of achievement appropriate functional parity between technical and biological systems (within the boundaries of general class of solving tasks).

Achieved the analogy of the structure of the "inner word" of internal image language with the structure of "inner word" of human language is the basis of identity (towards biological prototype) of accepted method of organization of information processes for determination of physical situations in the system "B - O" and prognosis capture reliability of non-oriented objects with estimations of physical situations, decision taking and the ability to control physical situations (in case of negative results of prognosis).

\subsection{Some Principles of Creation of Technical Thinking Systems (in our case)}

1. The principle of compatibility vectors, for example, of the main active forces with vectors of variable stiffness of the mechanism of brush of robot to ensure unstressed adaptation the brushes of robotics to shapes and positions of non-oriented objects.

2. The principle of similarity of the structures of language $\mathrm{s}$ (of robot and man, for example) based on modeling of method of organization of human thinking processes for intellectualization any thinking processes of robot.

3. The principle of prognosis the capture reliability of non-oriented objects as a steady state equilibrium of all forces and moments in the systems "Brush - object".

4. The principle of autonomy to ensure the determination of any physical situation in the system B-O and cognitive analysis of it.at autonomous level.

5. The principle of self-organization at structural level of robotics to ensure the search for acceptable solutions in case of any negative prognosis of capture reliability. 
Above represents the essence of technical functional analogues of human information processes in natural uncertain environment during the performing the same tasks.

\section{Robot "Cubic"}

As another example it is the domestic robot "Cubic"(Russia) [7] which is deprived any feet and hands and is capable to help its owner by need informational support. "Cubic" is able to understand and to apply a verbal human speech (Russian and English languages) in interactive mode and is ready to be a reviewer, an assistant, a secretary, a consultant and a coach.

Robot is a clever person to have a talking by using some aphorisms and humor, can help to set tasks and to take decisions, to read aloud last news, actual information, to provide remove control of any technical system of smart home or smart office.

\section{Practical Signification of Research}

Practical signification of research is following:

- recommendations for design of adaptive capture devices of robotics and intellectual prosthesis for invalids,

- recommendations for application of adaptive capture devices of robotics and intellectual prosthesis in natural conditions of human habitation,

- in Space - by creation of new special manipulators with capture reliability prognosis and variable lengths and lines of grippers for extra activities of astronauts in carrying out research, mechanical assembling, repair, rescue of constructions without changing the brushes,

- in Oceans - by participation in technology of deep-see non-diving rescue strap works on sunken vessels based on using the deep microappatures as type "Gnom", "Falcon", participation in clearance with shock-free capture mines and shells and putting them into the boxes,

- in Medicine - with creation of intellectual brushes, new prostheses devices and smart gloves of exoskeletons for functional rehabilitation of invalids, including with paralyzed hands,

- in Industry - by applications of automation and mechanization of technological processes in different small-scale and quick-skilled industries, for example, in aircraft, without changing the grippers, and so in machining, sheet metal stamping, casting, non-destructive inspection methods, including x-ray, and so on,

- in Sport by organization of new form of competition for building and remote original constructions by using manipulators with manual control and equipped with adaptive grippers and changed tools,

- in Domestic Sphere - by universal modules of household robotics, cleaning dishes and etc.

The result of the application of principles and methods of Technical Thinking Systems in manipulation robotics there is a real possibility of a large-scale invasion of manipulation robotics in an unknown and inaccessible earlier sphere of human activities as direct executers for manual and mechanized operations.

\section{Conclusions}

Above can stimulate as a promising large-scale replacement of working persons by future manipulation robotics in natural non-determinate environments, and so the improvement of robotics in the field of intellectualization, which in turn generates the analogy with the active role of a human hand in the material and spiritual spheres as the steps in the evolutionary processes of our Civilization and of Humanity itself.

\section{REFERENCES}

[1] A.I.Timofeev, V.A. Dmitrieva. The system of decision taking in indeterminate situations. B.S. Laboratory $-2^{\text {nd }}$ International Symposium "Systems Thinking for a Systainable Economy", Universitas Mercatorum, Rome, Italy. 23-24 January, 2014

[2] Колгоморов А.Н. Жизнь и мышление как особые формы существования материи. Из сборника “О сущности жизни”, М, Наука, 1964, с.52.

[3] Тимофеев А.И. Семиотическая основа процессов прогнозирования в неопределенных условиях. Материалы Десятой национальной конференции по искусственному интеллекту. 25-28 сент. Обнинск. Физматлит.2006.

[4] A.Timofeev. Artificial intellectual hand: Capture reliability prognosis of non-oriented complex shape objects for manipulating robotics. EMCSR 2012 - European Meeting on Cybernetics and Systems Research, University of Vienna, Austria, 10-13 of April, 2012.

[5] A.Timofeef, V.Dmitrieva. Civilization and technological thinking systems. $9^{\text {Th }}$ Congress of the UES-EUS. Globalization and Crisis. Complexity and Govermance of systems. Universitat Valencia, Spain, 15-16 October, 2014.

[6] Anatoly Timofeev. Civilization and Technical Thinking Systems. ISBN 978-3-659-67185-2, LAMBERT Academic Publishing, Germany, 2015, 42p..

[7] www.cubicrobotics.ru 